\title{
La eyaculación precoz. Actualización del tema
}

\author{
Darío Fernández Delgado
}

Médico de Familia, Psicólogo Clínico. Centro de Salud "Los Ángeles". Madrid. Correo electrónico: darioferdel@gmail.com

Recibido el 7 de noviembre de 2013.

Aceptado para su publicación el 8 de enero de 2014.

\section{RESUMEN}

La eyaculación precoz (EP) es una disfunción sexual presente en cualquier edad, pero actualmente infradiagnosticada en atención primaria a pesar de su incidencia en la salud sexual y calidad de vida. Ni se cura ni desaparece con la edad ni con la frecuencia sexual, todo lo contrario. La evitación de la relación sexual o incluso la disfunción eréctil la complican. El tratamiento farmacológico, aunque fundamental, no es suficiente. El abordaje sexológico y psicológico, desde el punto de vista de la pareja, es esencial. La mujer se ha de convertir en una coterapéuta.

Este artículo hace una revisión bibliográfica sobre la definición del término, métodos diagnósticos, implicaciones en la pareja y abordaje terapéutico. Para ello se han consultado los artículos originales publicados en castellano e inglés desde 2006 a 2013 en las bases de datos Pubmed y Cochrane.

No existe una definición única de EP. Las definiciones actuales del DSM muestran un bajo valor predictivo positivo y alto de diagnósticos falsos positivos. Todas las definiciones inciden en el tiempo de penetración vaginal. Existen varios cuestionarios estandarizados para el diagnóstico. Aunque hay variedad de ofertas de tratamiento, desde anestésicos locales pasando por cirugía, en España el único fármaco con indicación específica es la dapoxetina y ha demostrado su eficacia.

En conclusión, la EP es la disfunción sexual más frecuente en el varón, por encima de la disfunción eréctil. El diagnóstico y abordaje terapéutico es posible desde la consulta de atención primaria y exige un abordaje farmacológico y sexológico.

PALABRAS CLAVE: Eyaculación precoz, disfunción sexual, diagnóstico, atención primaria de salud.

\section{ABSTRACT}

\section{Premature ejaculation. Topic updates.}

Premature ejaculation $(\mathrm{PE})$ is a sexual dysfunction present at any age but currently underdiagnosed in Primary Care centers despite its high incidence on sexual health and quality of life. It does not improve or disappear with age or sexual frequency, completely the opposite. Avoiding sexual relations or even erectile dysfunction can make it worse. Pharmacological therapy, although essential, is not sufficient. A psychological and sexological approach, from the couple's point of view, is fundamental. The female partner should become a co-therapist.

This article provides a bibliographic review of the definition of the term, diagnosis methods, implications for the couple and therapeutic approach. To achieve this aim, the original articles published in Spanish and English from 2006 to 2013 in Pubmed and Cochrane databases have been consulted.

There is no single definition of PE. Current DSM definitions show a low positive predictive value and a high value in false-positive diagnosis. All definitions are centered on the duration of vaginal penetration. There are several standardized questionnaires for diagnosis. Although there is a wide offer of therapies, from local anaesthetics to surgery, in Spain the only drug specifically indicated for PE is dapoxetine and has proven its effectiveness.

In conclusion, PE is the most frequent sexual dysfunction in males, above erectile dysfunction. Its diagnosis and therapeutic approach is possible from the primary healthcare centers and requires a pharmacological and sexological approach.

KEYWORDS: Premature ejaculation, sexual dysfunction, diagnostic, primary health care. 


\section{INTRODUCCIÓN}

Los cambios sociales acaecidos ya en el pasado siglo han supuesto un protagonismo proactivo de la mujer en las relaciones sociales y, por ende, en las sexuales. La mujer exige una relación sexual de calidad y con la duración suficiente para alcanzar el orgasmo, de forma que la eyaculación del varón ahora ya ha de adaptarse y tener en cuenta la fase de respuesta sexual de la mujer, mucho más lenta. Si no es así, la salud sexual de la pareja y la convivencia se pueden resentir gravemente.

No se sabe con exactitud la causa de la eyaculación precoz (EP). Una primera experiencia sexual negativa y con prisas, una relación con una prostituta, la práctica habitual de la masturbación con miedo a ser descubierto en la adolescencia o la inexperiencia sexual aparecen descritos como antecedentes habituales. Pero también hay muchos eyaculadores precoces, expertos amantes sin estos antecedentes.

Se describen varios tipos de eyaculadores, pero todos coinciden en la incapacidad de controlar el momento de la eyaculación que ellos viven con ansiedad y ellas con insatisfacción. Esta disfunción no la cura el tiempo ni la edad, ambos lo complican con conductas de evitación sexual y hasta disfunción eréctil.

La eyaculación precoz es menos consultada que la disfunción eréctil. Hay que preguntar por ella en la consulta de atención primaria, desde aquí se puede tratar o derivar. Hay medios para hacerlo desde enfoques sexológicos y desde el imprescindible farmacológico. En este artículo se pretende hacer una revisión bibliográfica de artículos originales sobre la eyaculación precoz publicados en castellano e inglés durante el periodo 2006-2013 y utilizando como base de datos Pubmed y Cochrane.

\section{CONCEPTO DE EYACULACIÓN PRECOZ}

La EP es una disfunción sexual en la que el varón eyacula antes de lo deseado, a veces nada más iniciar el acto sexual, al tocar la vagina o incluso antes de intentar la penetración. El tiempo de latencia intravaginal hasta la eyaculación (IELT) que se considera promedio está entre 3 y 6 minutos. Se considera una eyaculación como precoz si se produce antes de un minuto y una EP probable si el IELT está entre 1 y 1,5 minutos.

Las asociaciones y organizaciones científicas han propuesto varias definiciones de eyaculación pre$\mathrm{COz}$, todas centradas en el control sobre el tiempo antes de eyacular aunque también consideran la percepción del grado de control sobre el reflejo y las consecuencias negativas para la relación de pareja.

Para la Asociación Americana de Urología el tiempo es el criterio decisivo y la define como "la eyaculación que ocurre antes de lo deseado, ya sea antes o poco después de la penetración, causando angustia a uno o a ambos compañeros" ". La Clasificación Internacional de Enfermedades-10 (CIE10), basándose en la capacidad de satisfacción sexual, la define como "la incapacidad de retrasar la eyaculación lo suficiente como para disfrutar de las relaciones sexuales, lo que se manifiesta por la aparición de la eyaculación antes o muy poco después del comienzo de la penetración, o se produce la eyaculación en ausencia de la suficiente erección como para que sea posible la penetración" 2 .

La Sociedad Internacional de Medicina Sexual (ISSM) incluye en la definición las consecuencias negativas sobre el varón. Propone, centrándose en una relación sexual clásica de varón-penetración vaginal, la siguiente definición: "disfunción sexual en la que la eyaculación se produce siempre o casi siempre antes o aproximadamente un minuto después de la penetración, debido a la incapacidad para retrasar la eyaculación en todas o casi todas las penetraciones vaginales y con consecuencias personales negativas, como angustia, malestar, frustración y evitación de las relaciones íntimas" 3.

El DSM-V propone la siguiente clasificación:

- Eyaculación Precoz Permanente, cuando ocurre antes de un minuto. Es desde siempre y probablemente es de causa genética o neurobiológica.

- Eyaculación Precoz Adquirida. Puede ser psicológica o somática. El varón refiere un momento biográfico claro de inicio.

- Eyaculación Precoz Natural Variable. Sólo en determinadas ocasiones se produce. Puede diagnosticarse como una variante normal.

- Disfunción Eyaculatoria Prematura que incluye a los eyaculadores considerados normales, de 3 a 7 minutos, incluidos los que duran más de diez minutos. Estos son los varones que se quejan subjetivamente de una eyaculación precoz, a pesar de la normalidad de sus tiempos. El origen es claramente psicológico.

La CIE-11 aporta un nuevo paradigma sindrómico, centrándose más aún en el IELT, basándose en estudios epidemiológicos en los que se ha cronometrado este tiempo. Aunque la eyaculación precoz 


\section{COMORBILIDAD}

Hipertiroidismo ${ }^{7}$

Infertilidad pareja ${ }^{8}$

Bajos niveles de prolactina y altos de hormona

tiroidea $^{9}$

Varicocele ${ }^{10}$

Disfunción eréctil ${ }^{11}$

Tabla 1. Comorbilidad asociada a eyaculación precoz

\begin{tabular}{|c|c|}
\hline Aspecto & Preguntas guía \\
\hline Diagnóstico & $\begin{array}{l}\text { ¿Cuánto tiempo pasa entre la penetración y la eyaculación? } \\
\text { ¿Se siente capaz de retrasar la eyaculación? }\end{array}$ \\
\hline \multicolumn{2}{|l|}{ Tipo de Eyaculación precoz } \\
\hline Primaria & ¿Cuándo experimentó la EP por primera vez? \\
\hline Secundaria & ¿Ha tenido EP desde su inicio sexual y con casi todas sus parejas? \\
\hline Consecuencias en la pareja & $\begin{array}{l}\text { ¿Nota disconformidad en su pareja por su EP? } \\
\text { ¿Su pareja evade las relaciones sexuales? } \\
\text { ¿Su EP está afectando a su relación global de pareja? }\end{array}$ \\
\hline Calidad de vida & $\begin{array}{l}\text { ¿Evade usted la relación sexual por miedo a sentirse mal? } \\
\text { ¿Se siente ansioso, deprimido o avergonzado por su EP? }\end{array}$ \\
\hline Asociación a disfunción eréctil & $\begin{array}{l}\text { ¿Son sus erecciones suficientemente rígidas como para penetrar? } \\
\text { ¿Ha apurado la relación sexual por miedo a perder la erección? }\end{array}$ \\
\hline
\end{tabular}

Tabla 2. Preguntas guía para abordar la eyaculación precoz en la consulta de Atención Primaria (Cedrés ${ }^{14}$ )

primaria posee un carácter orgánico, también se le atribuye un componente psicológico, en muchos casos un trastorno depresivo mayor ${ }^{4}$.

\section{EPIDEMIOLOGÍA Y COMORBILIDAD}

La eyaculación precoz es la disfunción sexual más frecuente, afectando al $16-23 \%$ de los varones ${ }^{5}$.

En el estudio NHSLS, realizado en EEUU, la prevalencia aumentó desde el 30\%, entre los 18 y 29 años, hasta el $55 \%$, en hombres de 50 a 59 años. El sesgo metodológico empleado en este estudio, al proponer como respuesta solo la opción sí/no podría explicar el haber encontrado una prevalencia muy superior a la de otros estudios ${ }^{6}$.

El 43\% de los españoles padecen eyaculación precoz en algún momento de su vida según el Estudio Demográfico Español sobre la Eyaculación Precoz (DEEP 2009) realizado por la Asociación Española de Andrología, Medicina Sexual y Reproductiva (no publicado). Es más prevalente que la disfunción eréctil. Resulta significativo el dato de que sólo el $38 \%$ pensaban que debería consultarse al médico en estos casos.

La tabla 1 muestra la comorbilidad asociada a la eyaculación precoz ${ }^{7-11}$.

\section{ETIOLOGÍA}

Actualmente, la teoría más en boga es la que se refiere a la sensibilidad de los receptores de la serotonina $5-\mathrm{HT}_{1 \mathrm{~A}}$ y $5-\mathrm{HT}_{2 \mathrm{C}}$ a nivel central y al $5 \mathrm{HTT}$, que facilita su recaptación en la sinapsis y que es el blanco de los inhibidores selectivos de la recaptación de serotonina (ISRS).

Existen evidencias que sugieren que la EP primaria es una variable biológica determinada genéticamente, relacionada con la sensibilidad heredada de los receptores centrales de la $5-\mathrm{HT}^{12}$. 


\section{DIAGNÓSTICO}

Aunque el diagnóstico se basa en la información aportada por el paciente y/o la pareja, actualmente de dispone de varios cuestionarios estandarizados:

- EI PEDT (Premature Ejaculation Diagnostic Tool)

- El PEP (Premature Ejaculation Profile)

- El Cuestionario de salud sexual masculina sobre disfunción eyaculatoria (Male Sexual Health Questionnaire Ejaculatory Dysfunction (MSHQEjD)

- El test de eyaculación precoz de Gindin y Huguet. Es el más usado. Puntuaciones entre 30-40 puntos indican una eyaculación precoz importante que requiere tratamiento.

La Guía Europea de Urología, actualizada en 2009, propone las siguientes recomendaciones para el diagnostico:

a) Evaluar el IELT autocalculado por el paciente, el control percibido sobre el reflejo eyaculatorio, la ansiedad y la dificultad interpersonal que provoca.

b) Realizar un examen físico en la evaluación inicial de la EP para identificar afecciones médicas subyacentes asociadas $u$ otras disfunciones sexuales, en particular la disfunción eréctil.

c) No se recomienda realizar análisis de laboratorio o neurofisiológicos de rutina.

La tabla 2 muestra las preguntas guía que propone Cedrés para abordar los casos de EP en la consulta de atención primaria ${ }^{13}$.

\section{TRATAMIENTO}

La tabla 3 nos muestra un resumen de las principales alternativas terapéuticas en casos de eyaculación precoz.

\section{Tratamiento etiológico}

Debe tratarse el cuadro orgánico que está provocando la eyaculación precoz.

\section{Tratamiento farmacológico}

- Los anestésicos tópicos están en desuso. Pueden causar reacciones cutáneas y una excesiva hipoestesia en el pene y en la vagina. No hay estudios fiables con estos fármacos.
- Los antidepresivos tricíclicos, como la clorimipramina, empezaron a usarse a principios de los años 70. Resultaron efectivos, pero hoy día, debido a sus efectos secundarios, han caído en desuso.

- Los inhibidores de la fosfodiesterasa tipo 5 (IPDE-5) y bloqueadores alfa-1 adrenérgicos no han sido aprobados para el tratamiento de la EP ni en EEUU (Food and Drug Administration [FDA]) ni en la Unión Europea (Agencia Europea del Medicamento [EMEA]).

- Inhibidores selectivos de la recaptación de serotonina (ISRS). En 1994 Waldinger fue el primero en demostrar la eficacia de los ISRS en alargar el IELT.

La dapoxetina, un ISRS de acción corta, es el primer fármaco diseñado específicamente para el tratamiento de la eyaculación precoz a demanda en varones de 18 a 64 años y el único aprobado para tal uso por las agencias internacionales del medicamento. Actualmente está autorizada su comercialización en siete países de la Unión Europea (Finlandia, Suecia, Austria, Alemania, España, Italia y Portugal). Es un ISRS de acción corta, que ha demostrado inhibir de forma estadísticamente significativa el reflejo eyaculatorio a nivel supraespinal ${ }^{14}$. No necesita, a diferencia de otros ISRS, de un periodo de impregnación, por lo que no es necesario administrarlo a diario, sino a demanda, en una dosis inicial de $30 \mathrm{mg}$ de 1-3 horas antes del coito ${ }^{15}$. Ha resultado igualmente efectivo tanto en la eyaculación primaria como en la secundaria. Se elimina principalmente por vía urinaria en 24 horas, sin otras sustancias activas. Esta farmacocinética lo convierte en el fármaco ideal para el tratamiento a demanda. La eficacia de la dapoxetina ha sido probada en ensayos clínicos, controlando los siguientes parámetros: el tiempo de latencia intravaginal hasta la eyaculación (IELT), el perfil de eyaculación prematura (PEP) y la percepción del control sobre la eyaculación $(\mathrm{EP})^{16}$. Todo esto convierte a la dapoxetina en el fármaco que mayores datos aporta sobre la eficacia en el tratamiento de la eyaculación precoz.

- Tramadol. Sin los efectos secundarios de los ISRS. Retrasa la eyaculación inhibiendo la recaptación de norepinefrina y de serotonina. Administrado a demanda, en dosis 
de $50 \mathrm{mg}$ dos horas antes del coito, ha demostrado alargar el IELT muy significativamente, según el estudio llevado a cabo en casos de eyaculación primaria ${ }^{17,18}$. Usado a demanda ha mostrado muy pocos efectos adversos ${ }^{19,20}$.

\section{Terapia sexológica}

\section{A nivel individual}

- Ejercicios de relajación

- Ejercicios de Kegel, practicando la contracción y relajación de la musculatura bulbocavernosa e isquiocavernosa. Los estudios realizados han sido con pocos pacientes, pero confirman éxitos del $60 \%$ tras cuatro meses de practicarlos ${ }^{21,22}$.

- A estos ejercicios se ha añadido la electroestimulación del suelo pélvico para pro- vocar contracciones en la zona y fortalecer la musculatura e incrementar la discriminación de sus contracciones. Estudios publicados refieren una mejoría en el $65 \%$ de los casos tras un año de tratamiento ${ }^{23}$.

\section{A nivel de pareja ${ }^{24-26}$}

- Técnica de parada / arranque (stop/start). Mediante la masturbación en pareja, se para cuando la excitación es tan alta que se percibe la inminencia eyaculatoria hasta bajar la excitación y comenzar un nuevo ciclo. Después de cinco ciclos se permite la eyaculación. De esta manera el paciente aprende a reconocer las sensaciones preeyaculatorias para poder después controlarlas.

- Técnica de compresión del pene (squeeze). Cuando el paciente percibe que va a

I. TRATAMIENTO ETIOLÓGICO de la causa orgánica

II. TRATAMIENTO FARMACOLÓGICO

- Anestésicos tópicos

- Antidepresivos tricíclicos

- Inhibidores de la fosfodiesterasa tipo 5 (IPDE-5)

- ISRS (Dapoxetina)

- Bloqueadores alfa-1 adrenérgicos

- Tramadol

III. TERAPIA SEXOLOGICA
Individual
- Ejercicios de relajación
- Ejercicios de Kegel
- Electroestimulación del suelo pélvico
Terapia en pareja
- Técnica de parada/ arranque (stop/ start)
- Técnica de compresión del pene (squeeze)
- Penetración vaginal sin movimientos.
- Técnica cognitivo conductual de la excitación
- Eliminación de las distorsiones cognitivas
- Técnicas de focalización sensorial
- Modelo simple sin fármacos
- Modelo combinado
Terapia en grupo

Tabla 3. Principales alternativas terapéuticas en casos de eyaculación precoz 
eyacular, la pareja o él mismo, con los dedos pulgar e índice, se comprime la región balano-prepucial hasta que desaparece la sensación. Bajado el nivel de excitación se reanuda la relación. El ciclo se repite cuatro o cinco veces para terminar eyaculando.

- Penetración vaginal sin movimientos. La mujer encima sin moverse. El varón introduce el pene y aguanta el tiempo que pueda, familiarizándose con las sensaciones sin eyacular.

- Técnica cognitivo conductual de la excitación, en la que tiene gran importancia que la pareja se comunique sus fantasías sexuales, sus pensamientos, se cuide el escenario y la preparación de la relación, así como el grado de excitación que esto supone para cada miembro de la pareja.

- Eliminación de las distorsiones cognitivas sobre la relación sexual. Rosen señala ocho tipos de distorsiones cognitivas ${ }^{27}$.

- Técnicas de focalización sensorial. Entrenan al paciente a ir más despacio y en centrarse en las sensaciones placenteras que le produce su pareja mediante la estimulación corporal, dejando para el final la estimulación en el área genital.

- Francisco Cabello ${ }^{28}$ del Instituto Andaluz de Sexología y Psicología propone su propio modelo completo y exhaustivo en el que integra diferentes estrategias con dos modalidades:

- $1^{\circ}$. Modelo simple, sin fármacos, que se compone de las siguientes fases: erotización sexual focalizada en mejorar la comunicación sexual de la pareja y propone ejercicios de autoestimulación erótica, pasando después a otra de erotización orgásmica y por fin a una desensibilización intravaginal.

- 20. Modelo combinado. En el anterior modelo, en la fase de erotización orgásmica se introduce el tratamiento con ISRS.

\section{Tratamiento en grupos.}

El objetivo debería ser reestructurar cognitivamente la interpretación individualista de la eyaculación precoz con la que acude el varón en solitario a la consulta de atención primaria y enfocarla como un problema de relación sexual de la pareja, que afecta a los dos y por lo tanto la solución ha de venir desde la pareja. Son necesarios para aprender a hacer cambios en el tipo de relación sexual que han llegado a construir los dos: ampliar el guión sexual, mejorar la comunicación, derribar tabúes, así como ayudarse de los puntos propuestos en los tratamientos citados. En este sentido el autor de este artículo, en colaboración con el servicio de urología del Hospital Clínico de Madrid, está llevando a cabo un programa de intervención grupal con parejas que se halla en fase de captación de pacientes.

En definitiva, existen diferentes ofertas terapéuticas, la mayoría centradas en retrasar el reflejo de eyaculación. Muchas lo consiguen. No está claro que la pareja solo y exclusivamente nos esté demandando retrasar el cronómetro, alargar la duración. Seguro que su demanda también incluye incrementar la satisfacción, por lo que el objetivo irrenunciable con cualquier terapia que se emplee, debería ser conseguir que la pareja llegue a "reconstruir" otro tipo de relación sexual más satisfactoria para ambos.

\section{BIBLIOGRAFÍA}

1. Montague DK, Jarow J, Broderick GA, Dmochowski RR, Heaton JP, Lue TF et al. AUA Erectile Dysfunction Guideline Update Panel. AUA guideline on the pharmacologic management of premature ejaculation. J Urol. 2004;172(1):290-4.

2. World Health Organization. The ICD-10 classification of mental and behavioural disorders: diagnostic criteria for research. 1993. Disponible en: http://www.who.int/classifications/icd/en/GRNBOOK.pdf [consultado el 17-022014]

3. ISSM. ISSM announces new definition of premature ejaculation. International Society of Sexual Medicine Newsbulletin, 2007; 24: 6 .

4. Waldinger MD, Schweitzer $\mathrm{DH}$. Changing paradigms from a historical DSM-III and DSM-IV view toward an evidencebased definition of premature ejaculation. Part II--proposals for DSM-V and ICD-11. J Sex Med. 2006;3(4):693705.

5. Perelman MA. A new combination treatment for premature ejaculation: a sex therapist's perspective. J Sex Med. 2006;3(6):1004-12.

6. Broderick GA. Premature ejaculation: on defining and quantiying a common male sexual dysfunction. J Sex Med. 2006; 3 Suppl 4: 295-302

7. Cihan A, Demir O, Demir T, Aslan G, Comlekci A, Esen A. The relationship between premature ejaculation and hyperthyroidism. J Urol. 2009;181(3):1273-80.

8. Lotti F, Corona G, Rastrelli G, Forti G, Jannini EA, Maggi M. Clinical correlates of erectile dysfunction and prema- 
ture ejaculation in men with couple infertility. $\mathrm{J}$ Sex Med. 2012;9(10):2698-707.

9. Corona G, Mannucci E, Jannini EA, Lotti F, Ricca V, Monami $M$ et al. Hypoprolactinemia: a new clinical syndrome in patients with sexual dysfunction. J Sex Med. 2009;6(5):1457-66.

10. Lotti F, Corona G, Mancini M, Biagini C, Colpi GM, Innocenti SD et al. The association between varicocele, premature ejaculation and prostatitis symptoms: possible mechanisms. J Sex Med. 2009;6(10):2878-87.

11. Porst H, Montorsi F, Rosen RC, Gaynor L, Grupe S, Alexander J. The Premature Ejaculation Prevalence and Attitudes (PEPA) survey: prevalence, comorbidities, and professional help-seeking. Eur Urol. 2007 Mar;51(3):81623.

12. Giuliano F, Clément P. Serotonin and premature ejaculation: from physiology to patient management. Eur Urol. 2006;50(3):454-66.

13. Cedrés S. Reseña clínico terapéutica de la eyaculación precoz. 2011. Disponible en: http://www.plenus.com.uy/ docs/hombre/EP novedades tto 2011.pdf [Consultado el 18/02/2014]

14. Clément P, Bernabé J, Gengo P, Denys P, Laurin M, Alexandre $L$ et al. Supraspinal site of action for the inhibition of ejaculatory reflex by dapoxetine. Eur Urol. 2007;51:82532.

15. Modi NB, Dresser MJ, Simon M, Lin D, Desai D, Gupta S. Single- and multiple-dose pharmacokinetics of dapoxetine hydrochloride, a novel agent for the treatment of premature ejaculation. J Clin Pharmacol. 2006;46(3):301-9.

16. Martín-Aragón S. Eyaculación precoz. Novedades farmacológicas. Offarm. 2011;30(2):48-54.

17. Bar-Or D, Salottolo KM, Orlando A, Winkler JV; Tramadol ODT Study Group. A randomized double-blind, placebocontrolled multicenter study to evaluate the efficacy and safety of two doses of the tramadol orally disintegrating tablet for the treatment of premature ejaculation within less than 2 minutes. Eur Urol. 2012;61(4):736-43.
18. Khan AH, Rasaily D. Tramadol use in premature ejaculation: daily versus sporadic treatment. Indian J Psychol Med. 2013;35(3):256-9.

19. Wong BL, Malde S. The use of tramadol "on-demand" for premature ejaculation: a systematic review. Urology. 2013;81(1):98-103.

20. Safarinejad MR, Hosseini SY. Safety and efficacy of tramadol in the treatment of premature ejaculation: a doubleblind, placebo-controlled, fixed-dose, randomized study. J Clin Psychopharmacol. 2006;26(1):27-31.

21. La Pera G. Awareness of the role of the pelvic floor muscles in controlling the ejaculatory reflex: preliminary results. Arch Ital Urol Androl. 2012;84(2):74-8.

22. Pastore AL, Palleschi G, Leto A, Pacini L, lori F, Leonardo $\mathrm{C}$, Carbone A. A prospective randomized study to compare pelvic floor rehabilitation and dapoxetine for treatment of lifelong premature ejaculation. Int $\mathrm{J}$ Androl. 2012;35(4):528-33.

23. Ferrán García J, Puigvert Martínez A, Prieto Castro R. Eyaculación prematura. Rev Int Androl. 2010;8(1):28-50.

24. Seco Vélez K. Eyaculación precoz: manual de diagnóstico y tratamiento. Caracas, Madrid: Editorial Fundamentos; 2009.

25. Carmita H.N. Abdo. Treatment of premature ejaculation with cognitive behavioral therapy. En: Emmanuele A. Jannini EA, McMahon CG, Waldinge MD. Premature Ejaculation. From Etiology to Diagnosis and Treatment. Milan: Springer; 2013. p 213-220.

26. Perelman MA. A new combination treatment for premature ejaculation: a sex therapist's perspective. J Sex Med. 2006;3(6):1004-12.

27. Rosen RC, Leiblum SR, Spector IP. Psychologically based treatment for male erectile disorder: a cognitive-interpersonal model. J Sex Marital Ther. 1994;20(2):67-85.

28. Cabello F. Manual de sexología y terapia sexual. Madrid: Editorial Síntesis; 2010. 